\title{
EXTENDED CONSTANT PARTS OF BECKER-STARK'S AND SHAFER-FINK'S INEQUALITIES
}

\author{
YUSUKE NISHIZAWA
}

\begin{abstract}
In this paper, we give some inequalities which are extended constant parts of Becker-Stark's and Shafer-Fink's inequality.
\end{abstract}

\section{Introduction}

Becker-Stark's inequality is known as that: for $0<x<\pi / 2$, the inequality

$$
\frac{8}{\pi^{2}-4 x^{2}}<\frac{\tan x}{x}<\frac{\pi^{2}}{\pi^{2}-4 x^{2}}
$$

holds, where the constants 8 and $\pi^{2}$ are the best possible constants. Shafer-Fink's inequality is known as that: for $0<x<1$, the inequality

$$
\frac{3 x}{2+\sqrt{1-x^{2}}}<\arcsin x<\frac{\pi x}{2+\sqrt{1-x^{2}}}
$$

holds, where the constants 3 and $\pi$ are the best possible constants. Many mathematicians researched Becker-Stark's inequality [1], [2], [7], [9]-[12] and Shafer-Fink's inequality [3]-[6], [8], [13]-[15]. As it now, the inequalities were extended in different forms. In this paper, we give inequalities which are the extended constant parts of inequalities (1.1) and (1.2). Our main theorems in this paper are as follows.

Theorem 1.1. For $8<r<\pi^{2}$ and $(\pi / 2)\left(\left(\pi^{2}-r\right) /\left(\pi^{2}-8\right)\right)^{1 / 3}<x<\pi / 2$, we have

$$
\frac{\tan x}{x}<\frac{r}{\pi^{2}-4 x^{2}}
$$

Theorem 1.2. For $3<r<\pi$ and $((r-3) /(\pi-3))^{1 / 4}<x<1$, we have

$$
\arcsin x<\frac{r x}{2+\sqrt{1-x^{2}}} .
$$

Received Received September 14, 2015, accepted March 28, 2016. 2010 Mathematics Subject Classification. Primary 26D05.

Key words and phrases. Becker-Stark's inequality, Shafer-Fink's inequality, power series, monotonically increasing function, monotonically decreasing function. 


\section{Proofs of main theorems}

\subsection{Proof of Theorem 1.1}

From $(\pi / 2)\left(\left(\pi^{2}-r\right) /\left(\pi^{2}-8\right)\right)^{1 / 3}<x<\pi / 2$, we have

and

$$
\pi^{2}-\frac{8\left(\pi^{2}-8\right) x^{3}}{\pi^{3}}<r<\pi^{2}
$$

$$
\frac{r}{\pi^{2}-4 x^{2}}-\frac{\tan x}{x}>\frac{\pi^{2}-\frac{8\left(\pi^{2}-8\right) x^{3}}{\pi^{3}}}{\pi^{2}-4 x^{2}}-\frac{\tan x}{x}=\frac{G(x)}{x\left(\pi^{2}-4 x^{2}\right) \cos x},
$$

where

$$
G(x)=x\left(\pi^{2}-\frac{8\left(\pi^{2}-8\right) x^{3}}{\pi^{3}}\right) \cos x-\left(\pi^{2}-4 x^{2}\right) \sin x .
$$

Hence, it suffices to show that $G(x)>0$ for $0<x<\pi / 2$. We show two lemmas to prove Theorem 1.1.

Lemma 2.1. For $0<x \leq 1$, we have $G(x)>0$.

Proof of Lemma 2.1. By Taylor series, we have

and

$$
x-\frac{x^{3}}{6}+\frac{x^{5}}{120}>\sin x
$$

$$
\cos x>1-\frac{x^{2}}{2}+\frac{x^{4}}{24}-\frac{x^{6}}{720}
$$

for $0<x<\pi / 2$. Here, for $0<x<\pi / 2$, we obtain

$$
\begin{aligned}
G(x) & >x\left(\pi^{2}-\frac{8\left(\pi^{2}-8\right) x^{3}}{\pi^{3}}\right)\left(1-\frac{x^{2}}{2}+\frac{x^{4}}{24}-\frac{x^{6}}{720}\right)-\left(\pi^{2}-4 x^{2}\right)\left(x-\frac{x^{3}}{6}+\frac{x^{5}}{120}\right) \\
& =\frac{x^{3}}{720 \pi^{3}} f(x),
\end{aligned}
$$

where

$$
\begin{aligned}
f(x)= & 2880 \pi^{3}-240 \pi^{5}+46080 x-5760 \pi^{2} x-480 \pi^{3} x^{2}+24 \pi^{5} x^{2}-23040 x^{3} \\
& +2880 \pi^{2} x^{3}+24 \pi^{3} x^{4}-\pi^{5} x^{4}+1920 x^{5}-240 \pi^{2} x^{5}-64 x^{7}+8 \pi^{2} x^{7} .
\end{aligned}
$$

It suffices to show that $f(x)>0$ for $0<x \leq 1$. The derivative of $f(x)$ gives

$$
f^{\prime}(x)=4(g(x)+h(x))
$$

where

$$
g(x)=11520-1440 \pi^{2}-240 \pi^{3} x+12 \pi^{5} x-17280 x^{2}+2160 \pi^{2} x^{2}+24 \pi^{3} x^{3}-\pi^{5} x^{3}
$$


and

$$
h(x)=2400 x^{4}-300 \pi^{2} x^{4}-112 x^{6}+14 \pi^{2} x^{6} .
$$

First, we may show that $g(x)<0$ for $0<x \leq 1$. Here, we have

$$
\begin{aligned}
g(x) & =11520-1440 \pi^{2}+x\left(-240 \pi^{3}+12 \pi^{5}-17280 x+2160 \pi^{2} x+24 \pi^{3} x^{2}-\pi^{5} x^{2}\right) \\
& =11520-1440 \pi^{2}+x\left(-240 \pi^{3}+12 \pi^{5}+x\left(-17280+2160 \pi^{2}+\left(24-\pi^{2}\right) \pi^{3} x\right)\right) .
\end{aligned}
$$

From $24-\pi^{2}>0$, we have

$$
\begin{aligned}
-17280+2160 \pi^{2}+\left(24-\pi^{2}\right) \pi^{3} x & <-17280+2160 \pi^{2}+\left(24-\pi^{2}\right) \pi^{3} \cdot 1 \\
& \cong 4476.48
\end{aligned}
$$

for $0<x \leq 1$. Hence, we can get

$$
\begin{aligned}
-240 \pi^{3} & +12 \pi^{5}+x\left(-17280+2160 \pi^{2}+\left(24-\pi^{2}\right) \pi^{3} x\right) \\
& <-240 \pi^{3}+12 \pi^{5}+x\left(-17280+2160 \pi^{2}+\left(24-\pi^{2}\right) \pi^{3} \cdot 1\right) \\
& <-240 \pi^{3}+12 \pi^{5}+1 \cdot\left(-17280+2160 \pi^{2}+\left(24-\pi^{2}\right) \pi^{3} \cdot 1\right) \\
& =-17280+2160 \pi^{2}-216 \pi^{3}+11 \pi^{5} \\
& \cong 707.206 .
\end{aligned}
$$

Therefore, we have

$$
\begin{aligned}
g(x) & <11520-1440 \pi^{2}+1 \cdot\left(-17280+2160 \pi^{2}-216 \pi^{3}+11 \pi^{5}\right) \\
& =-5760+720 \pi^{2}-216 \pi^{3}+11 \pi^{5} \\
& \cong-1985.02
\end{aligned}
$$

for $0<x \leq 1$. On the other hand, since

$$
h(x)=2\left(\pi^{2}-8\right) x^{4}\left(-150+7 x^{2}\right)
$$

and $-150+7 x^{2}<0$ for $0<x \leq 1$, we have $h(x)<0$ for $0<x \leq 1$. Thus, we have $f^{\prime}(x)<0$ and $f(x)$ is strictly decreasing for $0<x<1$. From

$$
\begin{aligned}
f(x) & \geq f(1)=24896-3112 \pi^{2}+2424 \pi^{3}-217 \pi^{5} \\
& \cong 2934.73
\end{aligned}
$$

we can obtain $f(x)>0$ for $0<x \leq 1$.

Lemma 2.2. For $1<x<\pi / 2$, we have $G(x)>0$. 
Proof of Lemma 2.2. By Taylor series, we have

$$
1-\frac{1}{2}\left(x-\frac{\pi}{2}\right)^{2}+\frac{1}{24}\left(x-\frac{\pi}{2}\right)^{4}>\sin x
$$

and

$$
\cos x>-\left(x-\frac{\pi}{2}\right)+\frac{1}{6}\left(x-\frac{\pi}{2}\right)^{3}
$$

for $0<x<\pi / 2$. Here, for $0<x<\pi / 2$, we obtain

$$
\begin{aligned}
G(x)> & x\left(\pi^{2}-\frac{8\left(\pi^{2}-8\right) x^{3}}{\pi^{3}}\right)\left(-\left(x-\frac{\pi}{2}\right)+\frac{1}{6}\left(x-\frac{\pi}{2}\right)^{3}\right) \\
& -\left(\pi^{2}-4 x^{2}\right)\left(1-\frac{1}{2}\left(x-\frac{\pi}{2}\right)^{2}+\frac{1}{24}\left(x-\frac{\pi}{2}\right)^{4}\right) \\
= & \frac{(\pi-2 x)^{2}}{384 \pi^{3}} f(x),
\end{aligned}
$$

where

$$
\begin{aligned}
f(x)= & -384 \pi^{3}+48 \pi^{5}-\pi^{7}-1536 \pi^{2} x+192 \pi^{4} x-4 \pi^{6} x \\
& -3072 \pi x^{2}+192 \pi^{3} x^{2}+16 \pi^{5} x^{2}-6144 x^{3}+768 \pi^{2} x^{3}-16 \pi^{4} x^{3} \\
& -512 \pi x^{4}+80 \pi^{3} x^{4}+1024 x^{5}-128 \pi^{2} x^{5} .
\end{aligned}
$$

It suffices to show that $f(x)>0$ for $1<x<\pi / 2$. We have the derivative

$$
f^{\prime}(x)=4(g(x)+h(x)),
$$

where

$$
\begin{aligned}
g(x)= & -384 \pi^{2}+48 \pi^{4}-\pi^{6}-1536 \pi x+96 \pi^{3} x+8 \pi^{5} x \\
& -4608 x^{2}+576 \pi^{2} x^{2}-12 \pi^{4} x^{2}
\end{aligned}
$$

and

$$
h(x)=-512 \pi x^{3}+80 \pi^{3} x^{3}+1280 x^{4}-160 \pi^{2} x^{4} .
$$

First, we may show that $g(x)>0$ for $1<x<\pi / 2$. The derivative of $g(x)$ is

$$
\begin{aligned}
g^{\prime}(x) & =8\left(-192 \pi+12 \pi^{3}+\pi^{5}-1152 x+144 \pi^{2} x-3 \pi^{4} x\right) \\
& =8\left(-192 \pi+12 \pi^{3}+\pi^{5}+3\left(-384+48 \pi^{2}-\pi^{4}\right) x\right) .
\end{aligned}
$$

From $-384+48 \pi^{2}-\pi^{4} \cong-7.66808<0$, we have

$$
\begin{aligned}
-192 \pi & +12 \pi^{3}+\pi^{5}+3\left(-384+48 \pi^{2}-\pi^{4}\right) x \\
& >-192 \pi+12 \pi^{3}+\pi^{5}+3\left(-384+48 \pi^{2}-\pi^{4}\right) \cdot \frac{\pi}{2} \\
& =-768 \pi+84 \pi^{3}-\frac{\pi^{5}}{2}
\end{aligned}
$$




$$
\cong 38.7742 \text {. }
$$

Thus, $g^{\prime}(x)>0$ and $g(x)$ is strictly increasing for $1<x<\pi / 2$. Here, we can get

$$
\begin{aligned}
g(x) & >g(1)=-4608-1536 \pi+192 \pi^{2}+96 \pi^{3}+36 \pi^{4}+8 \pi^{5}-\pi^{6} \\
& \cong 431.576
\end{aligned}
$$

for $1<x<\pi / 2$. On the other hand, since

$$
\begin{aligned}
h(x) & =16 x^{3}\left(-32 \pi+5 \pi^{3}+80 x-10 \pi^{2} x\right) \\
& =16 x^{3}\left(-32 \pi+5 \pi^{3}+10\left(8-\pi^{2}\right) x\right)
\end{aligned}
$$

and $8-\pi^{2}<0$, we have

$$
\begin{aligned}
-32 \pi+5 \pi^{3}+10\left(8-\pi^{2}\right) x & >-32 \pi+5 \pi^{3}+10\left(8-\pi^{2}\right) \cdot \frac{\pi}{2} \\
& =8 \pi .
\end{aligned}
$$

Hence, $h(x)>0$ for $1<x<\pi / 2$. Thus, we have $f^{\prime}(x)>0$ and $f(x)$ is strictly increasing for $1<x<\pi / 2$. From

$$
\begin{aligned}
f(x) & >f(1)=-5120-3584 \pi-896 \pi^{2}-112 \pi^{3}+176 \pi^{4}+64 \pi^{5}-4 \pi^{6}-\pi^{7} \\
& \cong 1168.07
\end{aligned}
$$

we can obtain $f(x)>0$ for $1<x<\pi / 2$.

Proof of Theorem 1.1. By Lemmas 2.1 and 2.2, the proof of Theorem 1.1 is completed.

\subsection{Proof of Theorem 1.2}

We show the following lemma to prove Theorem 1.2.

Lemma 2.3. For $0<t<1$, we have

$$
2-t+15 t^{2}-5 \pi t^{2}-12 t^{3}+4 \pi t^{3}>0
$$

and

$$
1-15 t^{2}+5 \pi t^{2}>0 .
$$

Proof of Lemma 2.3. We set

$$
f(t)=2-t+15 t^{2}-5 \pi t^{2}-12 t^{3}+4 \pi t^{3}
$$


Then, since we have

$$
\begin{aligned}
f^{\prime}(t) & =-1+30 t-10 \pi t-36 t^{2}+12 \pi t^{2} \\
& =-1+2(-3+\pi) t(-5+6 t) \\
& <-1+2(-3+\pi) \cdot 1 \cdot(-5+6 \cdot 1) \\
& \cong-0.716815
\end{aligned}
$$

$f(t)$ is strictly decreasing for $0<t<1$. From $f(1)=4-\pi>0$, we have $f(t)>0$ for $0<t<1$. On the other hand, from $-15+5 \pi>0$, we have $g(t)=1-15 t^{2}+5 \pi t^{2}=1+(-15+5 \pi) t^{2}>1$.

Proof of Theorem 1.2. From $((r-3) /(\pi-3))^{1 / 4}<x<1$, we have

$$
(\pi-3) x^{4}+3<r<\pi
$$

and

$$
\frac{r x}{2+\sqrt{1-x^{2}}}-\arcsin x>\frac{\left((\pi-3) x^{4}+3\right) x}{2+\sqrt{1-x^{2}}}-\arcsin x=f(x) .
$$

It suffices to show that $f(x)>0$ for $0<x<1$. The derivative of $f(x)$ gives

$$
f^{\prime}(x)=-2+x^{2}-15 x^{4}+5 \pi x^{4}+12 x^{6}-4 \pi x^{6}+2 \sqrt{1-x^{2}}\left(1-15 x^{4}+5 \pi x^{4}\right)
$$

and we set $t=x^{2}$ then the function $f^{\prime}(x)$ is equal to

$$
-\left(2-t+15 t^{2}-5 \pi t^{2}-12 t^{3}+4 \pi t^{3}\right)+2 \sqrt{1-t}\left(1-15 t^{2}+5 \pi t^{2}\right) .
$$

From Lemma 2.3, we can consider the following logarithm function: for $0<t<1$,

$$
\begin{aligned}
g(t)=\ln (2 \sqrt{1-t}) & +\ln \left(1-15 t^{2}+5 \pi t^{2}\right) \\
& -\ln \left(2-t+15 t^{2}-5 \pi t^{2}-12 t^{3}+4 \pi t^{3}\right) .
\end{aligned}
$$

The derivative of $g(t)$ gives

where

$$
g^{\prime}(t)=\frac{t h(t)}{2(1-t)\left(1-15 t^{2}+5 \pi t^{2}\right)\left(2-t+15 t^{2}-5 \pi t^{2}-12 t^{3}+4 \pi t^{3}\right)},
$$

$$
\begin{aligned}
h(t)= & -181+60 \pi+297 t-99 \pi t-105 t^{2}+35 \pi t^{2} \\
& -135 t^{3}+90 \pi t^{3}-15 \pi^{2} t^{3}+180 t^{4}-120 \pi t^{4}+20 \pi^{2} t^{4} .
\end{aligned}
$$

We have derivative

$$
\begin{aligned}
\frac{h^{\prime}(t)}{\pi-3} & =-99+70 t+135 t^{2}-45 \pi t^{2}-240 t^{3}+80 \pi t^{3} \\
& =-99+70 t-45(\pi-3) t^{2}+80(\pi-3) t^{3}
\end{aligned}
$$




$$
\begin{aligned}
& <-99+70 t+80(\pi-3) t^{3} \\
& <-99+70+80(\pi-3) \\
& \cong-17.6726
\end{aligned}
$$

Therefore, $h(t)$ is strictly decreasing for $0<t<1$. From $h(0)=-181+60 \pi \cong 7.49556$ and $h(1)=56-34 \pi+5 \pi^{2} \cong-1.46613$. there exists a uniquely real number $t_{0}$ with $0<t_{0}<1$ such that $h\left(t_{0}\right)=0, h(t)>0$ for $0<t<t_{0}$ and $h(t)<0$ for $t_{0}<t<1$. Since we have $g^{\prime}(t)>0$ for $0<t<t_{0}$ and $g^{\prime}(t)<0$ for $t_{0}<t<1, g(t)$ is strictly increasing for $0<t<t_{0}$ and $g(t)$ is strictly decreasing for $t_{0}<t<1$. From $g(0)=0$ and $g(1+)=-\infty$, there exists a uniquely real number $t_{1}$ with $0<t_{1}<1$ such that $g\left(t_{1}\right)=0, g(t)>0$ for $0<t<t_{1}$ and $g(t)<0$ for $t_{1}<t<1$. Therefore, we can obtain a uniquely real number $x_{1}$ with $0<x_{1}<1$ such that $f^{\prime}\left(x_{1}\right)=0, f^{\prime}\left(x_{1}\right)>0$ for $0<x<x_{1}$ and $f^{\prime}\left(x_{1}\right)<0$ for $x_{1}<x<1$. Thus, $f(x)$ is strictly increasing for $0<x<x_{1}$ and $f(x)$ is strictly decreasing for $x_{1}<x<1$. From $f(0)=0$ and $f(1)=0$, we have $f(x)>0$ for $0<x<1$. This completes the proof of Theorem 1.2.

\section{References}

[1] C.-P. Chen and W.-S. Cheung, Sharp Cusa and Becker-Stark inequalities, J. Inequal. Appl. 2011, 136(2011), 6 pp.

[2] L. Debnath, C. Mortici and L. Zhu, Refinements of Jordan-Steckin and Becker-Stark inequalities, Results Math., 67(2015), 207-215.

[3] A. M. Fink, Two inequalities, Univ. Beograd. Publ. Elektrotehn. Fak. Ser. Mat., 6(1995), $48-49$.

[4] B.-N. Guo, Q.-M. Luo and F. Qi, Sharpening and generalizations of Shafer-Fink's double inequality for the arc sine function, Filomat, 27 (2013), 261-265.

[5] B. J. Malešević, An application of $\lambda$-method on inequalities of Shafer-Fink's type, Math. Inequal. Appl., 10 (2007), 529-534.

[6] B. J. Malešević, Application of $\lambda$-method on Shafer-Fink's inequality, Univ. Beograd. Publ. Elektrotehn. Fak. Ser. Mat., 8 (1997), 103-105.

[7] D. S. Mitrinović, Analytic Inequalities, Springer-Verlag, 1970.

[8] Y. Nishizawa, Sharpening of Jordan's type and Shafer-Fink's type inequalities with exponential approximations, Appl. Math. Comput., 269 (2015),146-154.

[9] Z.-J. Sun and L. Zhu, Simple proofs of the Cusa-Huygens-type and Becker-Stark-type inequalities, J. Math. Inequal., 7(2013), 563-567.

[10] L. Zhu, A refinement of the Becker-Stark inequalities, Math. Notes, 93 (2013), 421-425.

[11] L. Zhu, Sharp Becker-Stark-type inequalities for Bessel functions, J. Inequal. Appl. 2010, Art. ID 838740, 4 pp.

[12] L. Zhu and J. Hua, Sharpening the Becker-Stark inequalities, J. Inequal. Appl. 2010, Art. ID 931275, 4 pp.

[13] L. Zhu, New inequalities of Shafer-Fink type for arc hyperbolic sine, J. Inequal. Appl. 2008, Art. ID 368275, 5 pp.

[14] L. Zhu, On Shafer-Fink-type inequality, J. Inequal. Appl. 2007, Art. ID 67430, 4 pp.

[15] L. Zhu, On Shafer-Fink inequalities, Math. Inequal. Appl., 8 (2005), 571-574.

General Education, Ube National College of Technology, Tokiwadai 2-14-1, Ube, Yamaguchi 755-8555, Japan.

E-mail: yusuke@ube-k.ac.jp 